\title{
Gärten in Wien.
}

Im botanischen Garten blühen derzeit:

Acacia leucocephata Lin k. -- Acacin frondosa W ill d.-Acacia lineata. - Aloè depressa H a v. - Cereus flagelliformis $\not$. nothus $\mathrm{H}$ o $\mathrm{t}$. - Cyubidium aloéfolium $\mathbf{S}$ va $\mathrm{rtz}$ - Cyrtocleilum spec. - Cistus candidissimus D i $\mathrm{n}$ a I. - Carmichelia australis. - Correa speciosa major B o ot $\mathrm{h}$. - Erodium cuneatun. - Eupatorium adenophorum S p r. - Itex Dahoon W al t. - Melaleuca imbricata $\mathrm{L}$ i $\mathrm{k}$. - Melaleuea incana R. B r. Maxilaria Henchmanni $\mathrm{H}$ o 0 k. - Nolana atriplicifolia D 0 n. - Oxybaphus viscosus Herit - Pomaderis andromadxefolia A. C a n ing h. Dimelea incana. - Paulownia imperialis S i ebold. - Stylidium adnatum R. B r. - Sempervivum caespitosum S m. - Vestia lycioides Willd.

\section{Fittheilungen.}

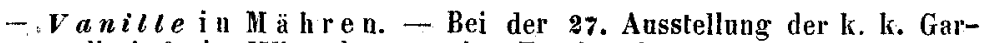
tenbaugesellscbaft in Wien lagen mehre Früchte der Vanille (Epidendrum Vamilla. L.) aus dem Garten des Grafen H ardeg.g zu Millotitz in Mähren vor. Diese Frïchte, welche vom Obergärtner $K u d l i c z k$ a durch künstliche Befruchtung im verflossenen Jahre zur Reife gebracht wurden, sind jedenfalls die ersten, welche man in Deutschland gewonnen hat.

- B a umgartuer's A l penha us. - Das wohlhekannte, im Jahre 1839 von dem Holzmejster Georg B a u ga $\mathrm{r}$ t $\mathrm{n}$ e auf dem Schneeberge erbaute Alpenhaus ist in der Nacht vom 24. - 25. November 1850 abgebrannt. Da es derzeit dem Besitzer dieses von allen Schneebergbesteigern besuchten Alpengasthauses unmöglich ist, dasselbe obne fremde Beihülfe zu erbauen; so erlässt der bekannte Tourist. F. C. W e i d m a $n \mathrm{n}$ in der Wiener Zeifung einen Aufruf an alle Freunde der valerländischen Alpenwelt um Beiträge zur Herstellung des Gebäudes für $B$ a um gartner. Solche übernimmt der Bäckermeister Herr Homan Uh I in seinem Verkaufsladen (Singerstrnsse Nr. 887). Das erste Verzeichniss der eingelaufenen Beiträge weist bereits eine Summe von 21 fl. 20 kr. CM. aus.

- Hortolog isches. -.-Die Engländer und Franzosen schmücken seit einiger Zeit einzelne, besonders feuchte, einsame, dumpfe und sonst halb verlorne Plätze ihrer Landschaftsgärten, mit eigenen Anlagen von Farrnkräıtergruppen, welche die Engländer fermery, die Franzosen fougeraie nennen, auch häufig an Grotten, Sturzwassern, Felspartieen anbringen. Natürlich nimmt man dazu nur die in Mittel - und Nord - Europa einheimischen Farrn Arten und bringt dazwischen reizende Gebilde von Kletten und Schlingpflanzen an.

- Upas. - Herr M a r chand, Marinelientenant auf der Flotte der vereinigten nordamerikanischen Staaten hat einen wegen seines Giftgehaltes bekannten Upas-Baum von Java nach New-York gebracht, Es gelang ihm, denselben in voller Vegetation auf der Ueberfalirt zu erhalten. Kurz var der Ankunft in No r folk. (Virginien), begann der Baum zu knospen und Blätter zu treibeu. Der Lieutenant hat ihn dem Nalionalinstitut zu Washington zum Geschenk gemacht, und derselbe ist im dortigen Observatorium zu seben. Alles, was von der gifthauchenden Atmosphäre dieses Vegetabils erzählt wurde, hat sich bei diesem Transporte, so wie bei früher augestellten Versuchen, als gänzlich falsch erwiesen. Bloss der Saft ist giftig und bringt schnell tödtliche Wirkung hervor.

(New-York Obst.)

- Oekon o mi sc hes. - Ein starker Hagelschlag hat bei B r es cia an vielen Orten die Hoffnung auf eine Ernte gänzlich vernichtet, so grossen Schaden hat er den Saaten gebracht. 